\title{
Global Non-State Actors (2020)
}

\author{
Tommaso Natoli*
}

In 2020, as expected, standard-setting activities of the main non-governmental actors actively engaged in the field of disaster risk management mostly dealt with the COVID-19 pandemic that gradually hit almost every country in the world over the year. This section will focus on the 'normative' activity led or supported by - three main NGOs clusters/networks: the Active Learning Network for Accountability and Performance in Humanitarian Action (ALNAP), the International Federation of Red Cross and Red Crescent Societies (IFRC), and Sphere. ${ }^{1}$

\section{The Active Learning Network for Accountability and Performance in Humanitarian Action (ALNAP)}

The Active Learning Network for Accountability and Performance in Humanitarian Action (ALNAP) is a global network of around 100 members (including NGOs, UN agencies, members of the International Red Cross and Red Crescent Movement, donors, academics and consultants) which has been active since 1997 towards the overall goal of improving the quality, availability and use of knowledge and evidence to make the humanitarian system perform better and be more accountable. ${ }^{2}$ In 2020 , this long experience was consolidated in a new guidance document for humanitarian agencies on 'Responding to coviD-19', based on a mix of desk-based research and key informants interviews. ${ }^{3}$ As evidenced by the words of Mark Lowcock, United Nations

* Irish Research Council/Marie Skłodowska-Curie Action CARoline Fellow in the School of Law of the University College Cork (UCC) and Research Consultant at the International Federation of Red Cross and Red Crescent Societies (IFRC).

1 For a theoretical reflection on the standard-setting activities of NSAS in this field, see Tommaso Natoli, 'Non-state humanitarian actors and human rights in disaster scenarios: normative role, standard setting and accountability', in Zorzi Giustiniani et al. (eds), 'Routledge Handbook on Human Rights and Disasters', (Routledge 2018).

2 ALNAP's activities are managed and implemented through its Secretariat, which is hosted by the Overseas Development Institute (ODI) in London.

3 Ben Ramalingam, Neha S. Singh, Audrey Mahieu and Karl Blanchet, 'Responding to COVID19. Guidance for humanitarian agencies (2020)', ODI/ALNAP - ALNAP Rapid Learning Review, available at $<\mathrm{https}$ //www.alnap.org/help-library/responding-to-covid-19-guidance -for-humanitarian-agencies>, last accessed (as any subsequent URL) on 15 April 2021. For 
Under-Secretary-General for Humanitarian Affairs and Emergency Relief Coordinator, the document "reflects the best available current knowledge on the implications of COVID-19 for humanitarian settings, and is a crucial resource for our community". ${ }^{4}$

To answer one central question - 'How should humanitarian organisations prepare and respond to COVID-19 in humanitarian settings in low- and middleincome countries?' - 14 key actions and 7 operational principles are outlined in the document. These are meant to represent leading elements for covidaffected humanitarian action in both conflict and disaster settings. One of the identified challenges was the fact that the majority of CoviD-19 response strategies have been designed for high-income countries and require, therefore, significant adaptations for use in such contexts.

Based on previous reports and databases on health emergencies, most of which were produced by the WHO, the 14 actions are split into five main categories, respectively dealing with:

1. Preparation of the health system - i.e. supporting national efforts to assess COVID-19 impacts and surge capacities; developing and communicating clear scenarios, guidance and protocols; and supporting integrated and coordinated decision-making and oversight,

2. Preparation of institutions and facilities - i.e. preparing across the continuum, from community to health facilities; prioritising people, money, essential services, and goods; and ensuring that PPE [personal protective equipment] for health workers and support staff is available,

3. Strengthening detection - i.e. prioritising syndromic facility-based surveillance; and adapting the testing approach to transmission scenarios and testing capacity,

4. Investing in prevention - i.e. with targeting that should account for and support the most vulnerable groups; employing containment approaches to reduce transmission; supporting socially and economically sensitive approaches to quarantine and isolation; and implementing WASH interventions for effective infection prevention and control, and

5. Enhancement of case management - adapting health facilities and protocols and delivering appropriate and relevant critical care measures.

A distinctive and positive feature of this list of actions is represented by the concise and pragmatic approach adopted, as in the case of the internal

the methodology aspects (with some divergences specified at 9) see Neil Dillon and Leah Campbell, 'ALNAP Lessons Papers: A Methods Note', oDi/ALNAP (2018) available at <https:// www.alnap.org/help-library/lessons-papers-a-methods-note $>$.

4 Ibid., 4. 
cross-references to different actions and some real case scenarios, charts and links to key resources.

The list of actions provided by the Guidance is also complemented by seven additional cross-cutting principles that should inform their implementation, and be considered in addition to the general humanitarian principles to be followed. These are widely informed by the need to combine top-down planning and anticipation with more bottom-up, community-led approaches and use a systematic, testable and adaptable approach to support and mobilise communities from the beginning. ${ }^{5}$ Indeed, the central role of national and local actors in the coviD-19 response is inevitable and needs to be supported by external humanitarian actors through harmonised and integrated efforts, including through "moving from a subcontractor mode of transferring risk to a partnership mode of sharing risks". ${ }^{6}$ Strengthening the political and social sensitivity of any response activities and establishing clear duty-of-care policies and protocols must be considered as essential criteria in this sense, while developing and agreeing on normative guidance on the ethics related to technical responses is essential to make sure that difficult decisions are made in the best possible way. ${ }^{7}$ The consideration of the so-called 'secondary impact' meaning other health-related as well as social and economic ones - is also addressed by one of the 'special' principles, and in particular, this implies developing and using a systemic lens to consider all of them. According to the guidance document, such a complex challenge can only be obtained through continuous investment in operational learning, research and innovation activities, as a necessary basis for effective evaluation and subsequent adaptive decision-making. This can be done in several ways, such as sharing available aggregated and disaggregated data by age, gender, ethnicity, displacement status and disability status or, where possible, enabling country-level research and learning strategies, broadening networks, prioritising engagement with relevant universities, business groups and trade associations at national and potentially regional level. ${ }^{8}$

5 Ibid., 44-45.

6 See reference to Ben Ramalingam, Bill Gray and Giorgia Cerruti, 'Missed opportunities: The case for strengthening national and local partnership-based humanitarian responses', ActionAid, CAFOD, Christian Aid, Oxfam GB and Tearfund (2013) available at <www.alnap .org/help-library/missed-opportunitiesthe-case-for-strengthening-national-and-local-par tnership-based $>$.

7 Ramalingam et al. (n 3) 46-48.

8 Ibid., 49-51. 

Societies

The International Federation of Red Cross and Red Crescent Societies (IFRC), the world's largest volunteer-based humanitarian network, coordinates the work of 192 National Red Cross and Red Crescent Societies worldwide. The IFRC acts before, during and after disasters and health emergencies to meet the needs and improve the lives of vulnerable people thanks to its volunteer network, relying on community-based expertise and following the principles of independence, neutrality, and impartiality as to nationality, race, gender, religious beliefs, class and political opinions. The IF RC also works to improve humanitarian standards and to persuade decision-makers to reduce vulnerabilities and strengthen resilience.

In 202O, as part of its mission, the IF RC supported National Red Cross and Red Crescent Societies' collective preparedness, containment, and mitigation activities for the CoviD-19 pandemic. This happened not only through the direct provision of funds, technical expertise and other resources but also through the development of technical guidance documents and other sets of recommendations. ${ }^{9}$ These ranged from the 'COVID-19 Planning Guide for Adapting Risk Communication and Community Engagement as Public Health and Social Measures Shift' (co-developed with Save the Children and the Johns Hopkins Center For Communication Programs) $)^{10}$ to the 'Financial Sustainability Guide and Toolkit for CoviD-19..11 As part of this effort, in May 2020 IFRC issued a document entitled 'Key Policy Issues for CoviD-19', providing a list of specific recommendations for governments, donors and the international community on how to work together to promote an effective public health response and ensure dignity for affected communities. ${ }^{12}$ The importance of addressing the 'secondary impacts' of the pandemic, including providing essential health services to keep treating non-COvid health issues,

9 See resources available at $<$ https://covid.ifrc.org/\#platforms $>$, and $<$ https://preparecen ter.org/site/covid-19/>.

10 The document is an initiative of the GOARN Risk Communication and Community Engagement (RCCE) Coordination Working Group co-led by UNICEF, the International Federation of Red Cross and Red Crescent Societies (IFRC) and the World Health Organization (wHO), available at <https://volunteeringredcross.org/wp-content/up loads/2020/o7/COVID-19-Planning-Guide-for-Adapting-Risk-Communicationand-Community-Engagement-as-Public-Health-and-Social-Measures-Shift.pdf $>$.

11 See $<$ https://volunteeringredcross.org/wp-content/uploads/2020/o7/o-2.-Guidance-for -NS-Financial-Sustainability_verı_rev.pdf $>$.

12 See $<$ https://volunteeringredcross.org/wp-content/uploads/2020/05/Handout-Policy -Issues-for-COVID-1-May.pdf>. 
was one of the elements stressed in the document. This includes, for instance, the need to meet people's mental health and psychosocial needs, which can be particularly difficult for those with pre-existing conditions. A similar understanding of specific needs that may be neglected is required for the most frequently marginalised groups, such as those living in slums, the socially isolated (especially older people), those with disabilities, in detention, in unsafe home environments with a high risk of violence, abuse or neglect, and those discriminated against due to their ethnicity or gender. In light of this, the policy recommends that " $[\mathrm{g}]$ overnments and other responders should engage with any potentially marginalised groups in order to design measures to meet their specific needs" and give particular consideration to the risks faced by migrants, refugees and other displaced persons to be excluded from pandemic prevention and support programmes. Indeed, border closures implemented in many countries can be viewed as an infringement of the right to non-refoulement, without a corresponding benefit to public health. Other recommended practices dealt with the protection of emergency responders, both staff and volunteers, to which governments and the humanitarian community must ensure access to training, appropriate personal protective equipment (PPE) and psycho-social support in case of need. According to the document " $[t]$ hose who fall ill in the course of their activities should be ensured coverage for their medical needs and, in case of death, compensation for their families".

Specific attention was devoted to the measures that governments should adopt with regards to their respective National Red Cross or Red Crescent Society, which should be provided with the necessary exemptions from control measures, such as movement restrictions, subject to appropriate precautionary and safety measures. These "should be included in national response planning and coordination, and authorities should feel free to call on their assistance in humanitarian activities", while at the same time "they should not be pressed to take up activities that go beyond their mission or their capacities or that might compromise adherence with the Fundamental Principles". This is in line with the call for external actors and donors to support the leadership of local actors and favour the inclusion of local civil society organisations in relevant coordination mechanisms, as well as equal partnerships based on respective capacities. At the same time, the international community is urged to keep providing support for "other humanitarian crisis", considering that the pandemic can render the provision of aid more challenging, dangerous and expensive. In this regard, a special mention is made to the need to continue identifying future climate and weather-related risks, in a complementary fashion to COVID preparedness and response activities. 
An important contribution provided by IFRC Disaster Law was the "Law and Public Health Emergencies Research Project". This Project, which was catalysed by the COVID-19 pandemic, aims to identify the key features of an effective domestic legal and policy framework for public health emergency (PHE) preparedness and response. The project involved country-level desktop research undertaken in two stages. The first stage of research was a mapping of COVID-19 emergency decrees in 113 countries during the initial stages of the pandemic. ${ }^{13}$ Thanks to pro bono support provided by legal professionals from all over the world, and based on publicly available materials, these mappings provide a relevant point of reference for identifying and researching the adoption of legal instruments at the national level during the COVID-19 pandemic. The mappings were prepared using a standard set of questions concerning, for instance, the coordination between state and non-state actors including the Red Cross/Red Crescent National Society; the responsibility ascribed to such actors; exceptions to travel restrictions, quarantine requirements, curfews, or other conditions; special legal facilities or exemptions for the importation of medical aid or other relief items or personnel, or exemptions to restrictions on business operations and opening hours; or measures concerning "vulnerable" groups.

The second stage of research, which involved analysis of legal and policy instruments used to respond to outbreaks and epidemics of other diseases (e.g. Ebola virus, SARs, MERs and Zika virus), involved a detailed assessment of domestic legal and institutional frameworks for public health emergencies in 33 countries. The questions posed in this stage focused, inter alia, on ascertaining the relationship between the legal and institutional frameworks for public health emergencies and other types of disasters, noting that the Bangkok Principles for the Implementation of the Health Aspects of the Sendai Framework recommend greater integration of health into disaster risk management and vice versa. ${ }^{14}$ Questions were also posed concerning the role of law and policy in mitigating secondary impacts of PHEs (including impacts on housing and human mobility) and in ensuring the protection of vulnerable groups (including, but not limited to, migrants, older people and people

13 The list of documents is available at $<$ https://volunteeringredcross.org/en/english-human itarian-diplomacy-and-advocacy/>.

14 See the Bangkok Principles for the implementation of the health aspects of the Sendai Framework for Disaster Risk Reduction 2015-203o (2016) available at <https://www .preventionweb.net/english/professional/policies/v.php?id=48396\#: :text=The\%2O 'Bangkok\%2oPrinciples'\%2oplace\%2ostrengthened,disaster\%2oand\%2ohealth\%2o risk\%2omanagement>. 
at heightened risk of the relevant disease or illness). A report summarising the results of the research project and providing a suite of recommendations was published in April 2021. ${ }^{15}$

Following its launch in 1997, the Sphere Project has aimed at increasing the quality of humanitarian work in disaster response through the identification of common standards and the provision of guiding documents. Its Sphere Handbook, among the oldest initiative in the field of humanitarian standards and last updated in 2018, is founded on the key 'Core Humanitarian Standard on Quality and Accountability (CHS) ${ }^{16}$ and the Humanitarian Charter, the groundwork document stating the ethical and legal principles that inspired the creation and further activities of Sphere initiatives. ${ }^{17}$ These documents are based on the idea of identifying the nature and basic characteristics of the response that response all crisis-affected people have the right to expect in order to survive and recover with dignity. On this basis, new guidance documents have been issued to re-frame the application of these foundational tenets to the COvID-19 pandemic and provide technical advice on how individuals, communities and humanitarian actors can best respond to the outbreak. Being a public health emergency, three topics among those already addressed in the Sphere Standards were considered of particular importance: Health and Water, Sanitation, and Hygiene Promotion.

These connections are summarised in a comprehensive document focusing on how to apply the Sphere standards in coronavirus responses and that also includes recommendations based on the Core Humanitarian Standard and other similar initiatives setting up sectoral standards. ${ }^{18}$ According to this guidance tool, it can be noted how the response to the pandemic for Sphere and its partner standards (Humanitarian Standard Partnerships - H PS) ${ }^{19}$ should be framed through four critically interconnected aspects:

1. Addressing information - "[p] ]eople have the right to understand what is happening and to trust that the measures taken are in their own and the

\footnotetext{
15 Available at <https://disasterlaw.ifrc.org/media/3010>.

16 See $<$ https://corehumanitarianstandard.org/>.

17 See <https://spherestandards.org/humanitarian-standards/humanitarian-charter/>.

18 See <https://spherestandards.org/coronavirus/\#Download>.

19 Including: Cash Assistance, Inclusion of older people and people with disabilities, Education in Emergencies, Child Protection and Markets and Economic Recovery.
} 
community's best interest [through] clear, transparent and understandable information";

2. Dignity - [a] void that "[p]eople who are living with conditions associated with stigma or indeed those who fear they may be stigmatised for having the Coronavirus can be driven to hide the illness to avoid discrimination";

3. Community engagement - "listen to [communities, including women, children, older people, persons with disabilities and other often excluded groups] and understand perceptions, social norms and beliefs to avoid the spread of rumours and misinformation";

4. Don't forget other needs and others - "[f]ocusing on preventing the spread of the Coronavirus should not make us forget affected people's other needs, nor the long-term needs of the wider population". ${ }^{20}$

These aspects are reflected in the holistic and people-centred approach to humanitarian work that is championed by the Sphere standards and related foundational documents, based on the idea that affected communities must always be involved in shaping the response. The need for community engagement was particularly accentuated by the nature of the global health emergency that we are currently experiencing, for instance, the need to promote critical hygiene practice like hand-washing by the entire population. Technical standards on handwashing included in the Sphere Handbook are therefore referred to as they provide key indicators on the minimum quantity of essential sanitary items per Household (i.e. two 10-20 litre water containers per household, one for collection, one for storage; 250 grams of soap for bathing per person per month). ${ }^{21}$ Similarly, other standards on WASH in healthcare settings (standard 6); Health System Standards (1.1-1.3, 1.5) and Communicable diseases (2.1.1 to 2.1.4) are directly relevant and put in connection with the COVID pandemic. Interesting, attention is paid to social aspects and norms, as it must be understood how to work with communities in finding alternative forms of greeting to replace handshakes or the way meat and animals are handled in marketplaces.

Other specific components of previously published standard-setting documents are summarised in the Guidance and placed in the context of the Covid emergency, with a focus on the specific points that can be useful to address the pandemic, and with a concise explanation of how to take advantage of them in the current context. This is the case for the 'Core Humanitarian Standard'; the 'Humanitarian Inclusion Standards for Older People and People

20 Sphere, 'Applying humanitarian standards to fight COVID-19' (2020) 1.

21 Sphere Handbook, Chapter 5 on 'Water Supply, Sanitation and Hygiene Promotion', Hygiene promotion standard 1.2: Identification, access to and use of hygiene items (2018). 
with Disabilities', the 'Inter-Agency Network for Education in Emergencies Minimum Standards'; the 'Minimum Standards for Child Protection in Humanitarian Action'; and 'The Minimum Economic Recovery Standards'; and the 'Livestock Emergency Guidelines and Standards'. As part of this collection, it must be highlighted how a new, specific guidance document on Cash and Voucher Assistance (CVA) was issued in 2020 by the 'Cash Learning Partnership., ${ }^{22}$ This living document, based on resources shared by the same humanitarian practitioners, is intended to help organisations understand and prepare for likely impacts of COVID-19 on their work, to consider whether CVA is right for the contexts in which they operate, and - if so - the considerations at each stage of the programme cycle for how to deliver these safely and effectively. This is particularly relevant considering that "Poverty can fuel contagion, but contagion can also create or deepen impoverishment". ${ }^{23}$

While not related to COVID, a final update concerning Sphere is the release of a new resource that supports humanitarian practitioners implementing Disaster Risk Reduction (DRR) activities while keeping a standard-based approach in mind. This is the second of a series of 'thematic sheets' that compile references to a particular topic and explain the mutual relevance of Sphere and DRR activities, common principles and approaches anchored in both, as well as a focus on Sphere's technical chapters, thus offering additional guidance in that area. ${ }^{24}$ This is aimed at encouraging Sphere users to mainstream Disaster Risk Reduction into their work and highlight to DRR specialists how Sphere supports their work, including through direct references to priorities identified in the Sendai Framework. This tool is particularly relevant considering that, despite the strong links between the two, the Sphere Handbook does not explicitly mention DRR in its principles and standards.

22 CaLP, 'CVA in COVID-19 contexts: guidance from the CaLP network', Version 14 (9 July 2020), available at <https://www.calpnetwork.org/wp-content/uploads/2020/o3/ CaLP-summary-guidance-version-14-9-July-202o-English.pdf>.

23 Vidya Diwakar, 'From pandemics to poverty: the implications of coronavirus for the furthest behind', ODI (10 March 2020), available at <https://www.odi.org/blogs/16754 -pandemics-poverty-implications-coronavirus-furthest-behind?utm_campaign=1348895_ ODI\%2onewsletter\%2O20\%2OMarch\&utm_medium $=$ email\&utm_source=Overseas\%2O Development\%2OInstitute\&utm_country $=\& d \mathrm{dm}_{-} \mathrm{i}=4 \mathrm{O}_{2} \mathrm{~W}, \mathrm{SWTB}, 3 \mathrm{CCRP} 3{ }_{3} \mathrm{H}_{9} \mathrm{Z}_{5}, 1>$.

24 Available at <https://spherestandards.org/wp-content/uploads/Sphere-ThematicSheet -DRR-EN.pdf $>$. 